\section{THE AUTUMN SKY 1}

II.

$A^{N}$ $\mathrm{N}$ attempt was made in a preceding paper to point out the most remarkable features of the planets that are at present so attractive for telescopic inquiry. We will now proceed to pass in review a few of the more interesting sidereal objects in that part of the heavens that is well placed for the observer. It is needless to say that within our limits nothing more can be expected than a very scanty selection, for the use of inexperienced students, of some of the most conspicuous of the many hundreds of double stars and nebulæ that are within the reach of ordinary instruments. We begin with the constellation Hercules, because it is rapidly gliding away from us, and the search for our first object should be undertaken as early as possible in the evening, especially if we are unacquainted with its position. The possessors, of course, of equatorial mountings and divided circles will readily find anything in our selection from its assigned place; but we propose to give such instructions as may be serviceable with altazimuth stands, aided by any common map, which will be occasionally supplemented by small diagrams. These, it must be borne in mind, correspond with a neridian position, and must be inclined one way or other to represent objects lying east or west of it.

If then we wish to find without an equatorial, set to R.A. $16 \mathrm{~h} .37 \mathrm{~m}$., D.N. $36^{\circ} 4 \mathrm{r}^{\prime}$, the great globular cluster in the constellation Hercules, known as M(essier) I3, we must look out west-north-west for a large triangle, nearly equilateral, of 3 rd mag. stars $\beta, \delta, \zeta$, the left-hand angle of which at $\beta$ is nearest the horizon; at the other end of this side is $\zeta$; if we continue the line $\beta \zeta$ nearly as far again to the right, bending a little upwards, we come upon a similar star $\eta$, and between $\zeta$ and $\eta$, but rather nearer to the latter, our object is found. It is easily visible with the slightest telescopic aid, and discernible even without it in a clear dark sky. It will be instantly recognised as a round ball of misty light, which nearer the meridian would be of considerable brightness. It will not be favourably placed for examination; but those who have once caught sight of it will look out for it in a better position another year. At any time its resolution into stars will of course depend on the aperture; as this and the power are increased the mass will soon begin to sparkle, and the more brilliant points will rise out of the general haze; but it will require a large telescope to resolve it throughout. The great Copenhagen achromatic of II inches aperture in the hands of d'Arrest effected it with a power of 95. Its components, ranging according to Sir J. Herschel from ro or II to I5 or 20 mags., must be thousands in number: his father had supposed I4,000. The state of compression, he observes, indicates not much greater density at the centre. Outliers surround it in streaky masses and lines; and the ball, according to the Earl of Rosse, is intersected by three dark rifts confluent towards the centre, which I have perceived, as known objects, with my $9 \frac{1}{3}$-inch mirror. This is unquestionably the finest specimen of a globular cluster visible in our latitudes; and even when the eye has recovered from its first surprise, it is never weary of reverting to this wonderful object. It may well be called wonderful, even at the distance at which we have to contemplate it. But imagination fails utterly to grasp the magnificence of such a scene, could we be transported to a standpoint two or three of its own diameters distant; or could we penetrate to the heart of the resplendent mystery, flaming on every side with suns innumerable, and where shade would be unknown and impossible. But are those thousands upon thousands suns indeed? We only know that they possess the solar character of intrinsic light; yet that there is something peculiar in that light appears

$$
\text { I Continved from p. Io }
$$

by modern analysis, which finds the red end of their spectrum deficient; but as to their nature, or their magnitude, or their distance from us, or among themselves; whether they were formed as they are, or have been gradually aggregated through innumerable ages-of all this we know absolutely nothing, and nothing are we ever likely to know. Nor if, as it is natural to suppose, gravitation is an inseparable property of matter, can we conceive how that glorious accumulation can be permanent, or escape ultimate transmutation into a fresh form of existence by the final coalescence of its members. It has indeed been supposed that, under certain admissions as to proportionate distance and velocity, such a mass might be preserved in a permanent condition of rotation; but we are treading here too closely upon the impossible, and though all may continue sensibly unvaried for ages, yet a secret principle must be at work that will issue in a final catastrophe-the opening, it may be, of a new and more glorious existence. We beings of a day can but confess our ignorance and our nothingness in the contemplation of such an evidence of creative power and uncomprehended skill in what is but a minute speck to the keenest eye.

We should not leave this object without noting its beautiful configuration in a large field with neighbouring stars ; probably only an optical vicinity. Yet who after all can say which may be the nearest, now that it has been so clearly shown that there is but a precarious relation between apparent magnitude and actual distance?

While we are in this region we should make an attempt to see another remarkable, though less-known, cluster, M 92, R.A. I7h. $13 \mathrm{~m}$., Decl. N. $43^{\circ} 16^{\prime} .^{1}$ It may be found without circles, by patient sweeping some distance above, and to the left of, the last. Not equal in size to $M_{13}$, it is more compressed and more brilliant in the centre; "formosissimus" in' the Copenbagen telescope. It is a singular circumstance that its spectrum resembles that of $\mathrm{M}$ I3.

Next, ever-charming Lyra, with its glowing sapphire, Wega, the beauty of the northern sky, whose minute I I mag. attendant at about $46^{\prime \prime}$ is a well-known test for sensitiveness of vision. If this, as we are warranted in supposing, is a sun even more magnificent than our own, a search for a planetary system might not be hopeless. Several observers have actually seen minute points in its immediate neighbourhocd, but there is little agreement about them, and they remain for closer investigation. The accompanying diagram may serve to "guide us to

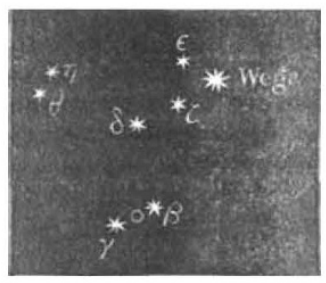

some other interesting objects here. Above Wega, a little to the left, are the two well-known pairs, $\epsilon^{1}, \epsilon^{2}$, with the intervening debilissima; long familiar to observers. It is no very uncommon feat, though one which I could never accomplish, to separate $\epsilon^{1}$ and $\epsilon^{2}$ at $3 \frac{1}{2}^{\prime}$ distance with the naked eye; the subdivision of the pairs, and the ruddier hue of one of the components of $\epsilon^{1}$, will be apparent with a power of 50 or 60 . There is of course an abstract possibility that this beautiful combination may be merely the result of coincident direction : but our optical sense revolts from the demand this would make upon it; and

I This glorious object, discovered by Bode, is omitted in Sir J. Herschel's Catalogue. 
mere inspection forces home upon us the persuasion that a physically-connected system Jies before the eye. As regards each pair separately, the inference is already established by orbital motion - the mutual relation of the whole waits the examination of ages.

The debilissima may be watched for a suspicion of variable light. Some very minute points precede them.

$\zeta$, next below and following $W$ ega, is a grand wide pair, nearly $44^{\prime \prime}$ apart, 5 and $5 \frac{1}{2}$ or 6 mags., topaz yellow and greenish, or, according to others, lilac. Such discordant estimates of colour are of frequent occurrence. They

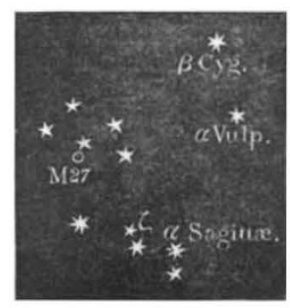

2.

may arise from actual dissimilarity of vision, or an uncertain effect of contrast, or residual chromatic aberration; but we have to remember that these star-hues are of ten open to question from their feebleness, and especially from the absence of comparison with a light of standard whiteness in the field.

To the left of $\zeta$ a low power will show us $\delta^{1}$ and $\delta^{2}$ in a fine group, where some beautiful colouring will be found. One of the smaller stars has at times appeared to me of a pale ruby tint, on other nights not traceable. 24' nearly north of $\delta^{1}$ Burnham bas detected a sma l pair (his No. 137), only $\mathrm{I}^{\prime \prime} \cdot 2$ apart, and therefore a severe trial for ordinary apertures.

Further to the left the naked eye shows us two small stars at a greater distance : the uppermost, $\eta, 5$ mag., has a 9 mag. attendant at $28^{\prime \prime}$; the colour is differently recorded as blue and pale yellow. Three small pairs lie near it. The other star, $\theta$, is also worth looking at for its fine surroundings. But beautiful fields are throughout more or less the character of this constellation.

Lower down, and brighter than the two last-mentioned, are two considerable stars, $\beta$ and $\gamma$, of which the right hand one, $\beta$, is the centre of a minute group; but more remarkable for variation in its light, with superposed inferior variations, completed in about $12 \mathrm{~d}$. $22 \mathrm{~b}$. Its

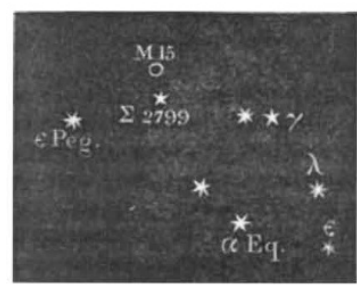

3.

colour is also questionable, yellow, or none. But greater interest attaches to these two stars as guides on either side to the wonderful annular nebula, M 57, the brightest of its class, easy with very slight optical means, refractory with the highest. Its light has been noticed, ever since Schröter's time, to be a little unequal in different parts, and may possibly be variable; the interior is gauzy and streaky, according to the Earl of Rosse, who also represents some exterior wispiness. The idea of its starry composition, maintained by Secchi and Chacornac, has been dispelled by Huggins, whose spectroscope shows only gas. But how produced? how accumulated into that extraordinary shape? how beated into incandescence, and maintaining that temperature, at any rate since 1779, amid the alleged intensity of cold in space-here even conjecture must admit its helplessness. A minute star following it may perhaps be variable.

In sweeping from this region towards the galaxy, we shall cross an abundance of rich and lovely fields; but

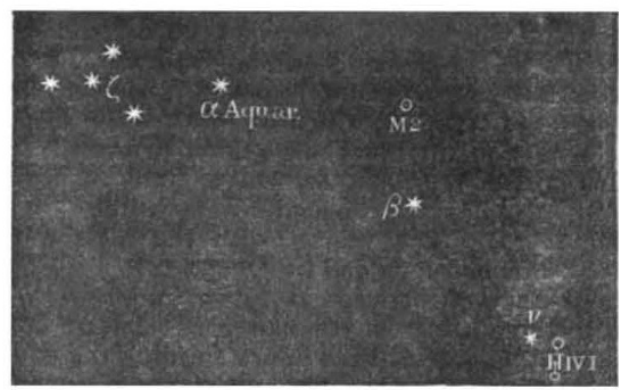

4.

the galaxy itself, with a sufficient aperture and low power, is from end to end a scene of wonder and astonishment. Especially in some parts of Cygnus its magnificence exceeds all hope of description. But it is not only to be studied for its gorgeous profusion of suns-where in a short time many thousands pass before the eye-nor for the many beautiful pairs that it envelopes-such as the

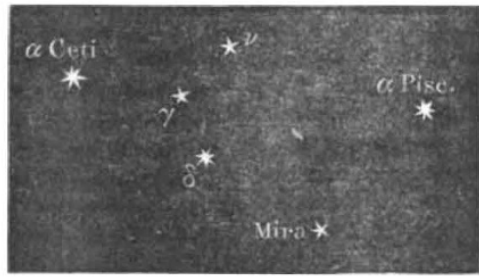

well-known $\beta$ Cygni, with its elegant contrast ; or $\chi$ (R.A. Igh. $42 \mathrm{~m}$. D.N. $\left.33^{\circ} 27^{\prime}\right)$, inferior indeed, but very pretty; or the wide pair 6 I (R.A. 2 Ih. Im. D.N. $38^{\circ} 9^{\prime}$ ), whose parallax, the first well established, shows that they are nearer to us than the bright leaders of the constellation; or at the end of the right arm of the great cross, $\delta$, whose minute comes at $1^{\prime \prime} \cdot 6$ has proved such an annoyance to many a

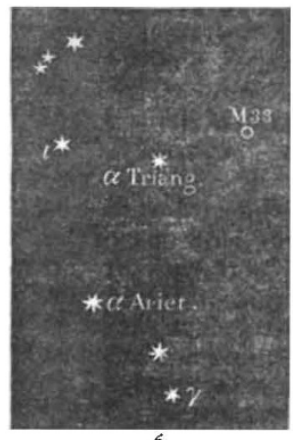

6.

disappointed observer, who might however have succeeded in a twilight hour; nor again for its groups of irregular and artistic beauty; but for many peculiarities of arrangement, not altogether unrecorded, especially by Secchi, but scarcely adverted to as they deserve. Among the minuter stars, from perhaps the 8th to the IIth mag., configurations are not infrequent of such singular 
regularity or peculiarity of arrangement that the idea of optical concurrence seems inadmissible. Triangles, squares, rhomboids, wreaths, festoons, coronets, indicate a collocation utterly inscrutable, but certainly not fortuitous. Such combinations are occasionally met with in other regions, but, as might be expected, are accumulated in the galaxy.

The glory of this sparkling zone, culminating in the splendours of Cygnus, is everywhere repeated in greater or less degree. Aquila, Sagitta, Vulpecula, Lacerta, to say nothing of more distant regions, all present fields of most gorgeous richness, which we must leave unnoticed. But before we finally cross this region to the other side, we must pause in Vulpecula, where, in R.A. $19 \mathrm{~h} .54 \mathrm{~m}$. D.N. $22^{\circ} 23^{\prime}$, lies an especial mystery, the Dumb-bell Nebula (M 27). The search with the altazimuth will prove not difficult as guided by Diagram 2 .

In mid-distance between $\beta$ Cygni and a Aquila we we shall recognise a lengthened group of 4 th and $5^{\text {th }}$ mag. stars, the lowest in the figure, forming the constellation Sagitta. We may remark, in passing, that one of these, $\zeta$, R.A. Igh. 44 m., D.N. $18^{\circ} 5 \mathrm{I}^{\prime}$, is a very pretty wide double, the largest of which has been found by Alvan Clark, jun., to be an excessively close pair. Nearer to $\beta$ Cygni, but to the left, is another less marked group, forming part of Vulpecula, close to one of the stars of which, in a rich field, we shall perceive two oval hazy masses in lateral contact, the nebula in question.

Like other objects of the same nature, this has been seen, or at any rate, drawn, in very dissimilar ways; it seems in fact almost inseparable from the character of nebulous light that its limits and gradations should be differently appreciated by different observers; and in this instance the additions resulting from larger apertures have almost obliterated the original idea; yet without suspicion of actual change. Minute twinklings are easily seen in the haze; and the Earl of Rosse finds a much greater abundance of stars than in the surrounding sky; yet he does not resolve it ; and the spectroscope shows it as a huge mass of incandescent gas-apparently the little. comprehended element nitrogen.

Quitting now the galaxy for the region on its eastern side, we notice, a little above $a$ Aquilæ, the familiar lozenge of Delphinus (a curious instance of configuration) the following or left-hand component of which, $\gamma$, is a noble pair of 4 th and 7 th mags. at nearly $12^{\prime \prime}$, and somewhat differing in colour now, though Sir W. Herschel called them both white in I779. Physical connection is inferred from an extremely slow relative movement; more decidedly from a motion through space common to both-an unsolved and insoluble mystery. South of $\gamma$, a little to the west is $\Sigma 2725$, a very pretty little couple, at $4^{\prime \prime} \cdot 2$; possibly of binary character.

A little further south-east we shall find another less conspicuous group of small stars, chiefly in the form of a trapezoid. This is Equuleus. It is given in Diagram 3, where $a$ is at the lowest angle.

Here $\gamma$, the $4 \frac{3}{4}$ mag. star nearest Delphinus, forms a striking combination with one of 6th mag., and has itself been doubled by Knott with an II mag. comes at $2^{\prime \prime} \cdot 1$. And beyond the limit of the trapezoid to the south-west we find a very fine triple, $\epsilon$, R.A. 2oh. 53m., D.N. $3^{\circ} 50^{\prime}$, the two closest of which are a beautiful pair in slow rotation, whose distance, less than $I^{\prime \prime}$, renders it an excellent test for a moderate aperture. The third star also appears to be physically connected with the others. $\lambda$, readily found by sweeping less than $3^{\circ}$ north of the last, and a little east, is also well worth looking for : 6 and $6 \frac{1}{2}$ mags. at about $2^{\prime \prime}$.

We shall now cross the border into Pegasus, the leader of which, in respect of position, $\epsilon$, is represented in the same diagram; a bright yellow $2 \frac{1}{2} \mathrm{mag}$. star with 2 comites, 9 and 14 mags. Sweeping rather more than $21^{\circ}$ north of this, about I4m. west (R,A. 2 rh. 24 m., D.N. II $^{\circ}$ $38^{\prime}$ ) we shall come upon M I5, a noble globular cluster, of similar character to M I3 in Hercules, but of smaller size. Sir J. Herschel gives the stars I 5 mag. of his scale, running up to a central blaze, and more condensed there than according to the idea of equal distribution. It is not quite circular, and surrounded with outlying streams. Buffham, with a 9-inch "With" mirror, has detected a dark patch near the centre, and two faint rifts like those in M I3, traces of one of. which I think I once perceived. This fine object deserves a long and steady gaze.

About $\mathrm{I}^{\circ}$ south of the cluster, a little west, we shall readily find a beautiful pair, perhaps slow binary, $\Sigma$ 2799, both 6.6 mag. of Struve's scale, $I^{\prime \prime} 4$ distant.

Ranging east and west, at some distance below $\in$ Pegasi, we shall remark the subject of Diagram 4; part of Aquarius. At the east end a very remarkable pair, $\zeta$, R.A. 22h. 23m., D.S. $0^{\circ} 38^{\prime}$, will be found in the interior of an easily-recognised triangle of stars, not much differing in brightness. The magnitudes are given 4 and $4 \frac{1}{2}$; the distance $3^{\prime \prime} \cdot 6$; the binary character is certain, and the period last assigned 1625 years. This is an excellent object for a small telescope, and will lead to many wondering thoughts as to the possible state of existence there. A much larger aperture will be required to secure Burnham's little pair, $8 \frac{1}{2}$ and Io or II mag. at $1^{H \prime} \cdot 5, \mathrm{II}^{\prime}$ south of $\zeta$.

To find our next object, M 2, R.A. 2 Ih. 27 m., D.S. $I^{\circ} 22^{\prime}$, by sweeping, we must note its configuration in the diagram with $a$ and $\beta$, two stars conspicuous for insulation in a dull region. It will repay the search, being, not indeed a brilliant, but a very interesting ball of several thousand very minute stars, $15 \mathrm{mag}$. of Sir J. Herschel, "a most superb cluster" in his reflector, "like a heap of fine sand," blazing in centre: D'Arrest sees it of irregular form. What an object, could we reach its neighbourhood!

But perhaps more surprising, though in quite a different way, is the next, HY iv. I-that is, No. I in Sir W. Herschel's fourth class - a noble specimen of what, from their equable light and defined edges, the illustrious discoverer termed Planetary Nebulæ. It lies in R.A. 2oh. $58 \mathrm{~m}$., D.S. $11^{\circ} 50^{\prime}$, and, to find it, we may carry a line through $a$ and $\beta$ nearly as far again, bending a little downwards : this will point out $\nu$, a 5 th mag. star, a little west of which we shall catch our object. It is tolerably bright, slightly elliptical, and of a pale blue tint. Lassell has seen a luminous well-defined ring in its interior; Buffham, with 9 -inch mirror, an opening. The Earl of Rosse, besides many faint projections, finds a narrow ray on either side, making the whole a singular resemblance to Saturn with a very thin presentation of the ring. Yet here too is gas! and as it seems, nothing but gas! But whence? how? wherefore?

And now we pass to the left over a long and rather dull region from the triangle in Aquarius to the chief star of Pisces, at the extremity of the constellation, about $20^{\circ}$ under the well-known three stars that mark the head of Aries. a Piscium with the head of Cetus east of it are shown in Diagram 5. It is a fine pair, 5 and 6 mags. at $3^{\prime \prime} \cdot 2$, the tints of which, as well as the mags., have been described with greater discordance than can be easily explained. a Ceti, the next conspicuous star to the east, is a very beautiful specimen of a large orange star, with a blue companion at a little distance, the colour of which may possibly be due to contrast, or heightened by it : this might be ascertained by hiding the great leader behind the edge of the field. There is a pretty little pair near them. The next star to the right, $\gamma$, is a striking double, $2^{\prime \prime} \cdot 6$ apart, 3 and 7 mags., yellow and blue (greenish, ruddy, or tawny to some eyes). Above and to the right of this is $\nu, 4 \frac{1}{2}$ mag., attended by a very minute companion, a glimpse star to Smyth, but much easier of late. Below $\nu$, a little to the right, is $\delta$, a line through which from $a$ will point out a very remarkable variable, $o$, 
or Mira Ceti, R.A. 2h. 13 m. D.S. $3^{\circ} 31^{\prime}$. This changes from 2 mag. to equality with a very minute companion in about 33Id. $8 \mathrm{~h}$. $4 \mathrm{~m}$., but not without some uncertainty. Its colour, according to Sir J. Herschel, is a full ruby ; I saw it so once, when about 10 mag., if it was the right star, but it was immediately clouded over : at another time, near maximum, pale yellow, without a trace of red. Flammarion also finds it by direct comparison less red than a gas flame. This tint would be an interesting object of study; but care must be taken in identification. Mira is now decreasing.

Having mentioned the head of Aries, we must recollect that $\gamma$, the smallest of the three stars, is a double, interesting as the first of these objects recorded by Hooke in 1664 , and a fine object, nearly $9^{\prime \prime}$ distant. These three stars are introduced into this diagram as guides to Triangulum just above them, where we shall find our last two objects.

', R.A. 2h. 5m., D.N. $29^{\circ} 44^{\prime}$, topaz and green, an exquisitely coloured pair, $5 \frac{1}{2}$ and 7 mags., distance $3^{\prime \prime} \cdot 5$; perhaps in slow orbital motion. We shall pick it up about $\frac{3}{4}^{\circ}$ north of $a$, a little to the east. The other is a strange phænomenon,

M 33, R.A. Ih. $27 \mathrm{~m}$., D.N. $30^{\circ} 4^{\prime}$, about $I^{\circ}$ north of $a$, and not far towards the west. Feeble as it is, it will be visible even with a very small aperture, from its great extent; fully $\frac{1}{2}^{\circ}$, or the diameter of the moon, from north to south; but on the other hand may, from want of contrast, be imperceptible with any but a very low power. The elder Herschel thought it was resolved into the minutest possible stars; but this has not been confirmed. His son and d'Arrest find a principal condensation with subordinate nuclei and nebulous tracts; the Earl of Rosse, a flocculent and spiral structure, with curved intersecting branches; a strange, incomprehensible form.

Our space will not admit of an extension of this very meagre and imperfect selection, limited for the most part, for obvious reasons, to such portions of our autumnal sky as are rapidly passing away.

T. W. WEBB

\section{AN OBSERVATORY FOR HONGKONG}

$\mathrm{W}^{\mathrm{E}}$ observe that Sir John Hennessy, the Governor of Hongkong, has succeeded in getting an item of twenty thousand dollars for an observatory and time ball passed by his Legislative Council in the estimates for the current year, in addition to a sum of ten thousand dollars voted for the same purpose last year. The advice of Major H. S. Palmer, of the Royal Engineers, has been sought by the Colonial Government, and in a valuable paper published in one of the most recent Government Gazettes, he details the advantages, imperial and local, which may be expected from a physical observatory in Hongkong. The favourable position of the island for certain observations, especially with regard to the typhoons of the China Seas, on meteorology generally, and on terrestrial magnetism, was first brought to the Governor's notice in September, I879, through the Secretary of State for the Colonies, by Dr. Warren De La Rue, as ViceChairman of the Kew Committee of the Royal Society. $\mathrm{He}$ pointed out the extreme importance of obtaining accurate records of the magnetic and meteorological conditions of the China Seas. There are at present only four observatories at which continuous observations are taken on the eastern coast of Asia and the adjacent islandsone at Batavia, in Java, supported by the Dutch Government, one attached to the Russian embassy at Peking, one at Siccawei, near Shanghai, and one at Manila. The two last are supported by the Society of Jesus. It is pointed out that Hongkong divides the distance between Siccawei and Manila, and would consequently be a most valuable acquisition to the list. The support of the Government of the Colony for a limited number of years to an observatory provided with self-recording instruments is therefore invoked. English officials and merchants abroad do not as a rule display much interest in science, and it is therefore fortunate that the gentleman at present at the head of the Government of Hongkong is one capable of appreciating the great importance of Dr. De La Rue's suggestion and of energetically carrying it out. Governor Hennessy had indeed in some sense anticipated the request, for he had, two years previously, in the first estimates which be prepared for the colony, obtained five thousand dollars from his Council for an observatory and time-ball. He entirely agreed with Dr. De La Rue's remarks, and added that the clearness of the atmosphere at certain seasons would admit of a valuable record being made of sun-spots, while the tides and varying temperature of the seas surrounding Hongkong, and the slight but frequent earthquake phenomena, would also be deserving of the attention of competent observers. A sum of between thirty and forty thousand dollars has been granted altogether for the establishment of an observatory, the chief objects of which are-

I. To determine the local time by astronomical observations, and drop a time-ball daily.

2. To obtain a series of meteorological observations with instruments of the best kinds, and to acquire information relating to the typhoons and monsoons of the China seas.

3. To obtain a series of observations in terrestrial magnetism, also with the best modern apparatus.

In his report Major Palmer addresses himself to each of these subjects separately. He recommends, we are glad to notice, that none but the best instruments should be employed, and that the observatory records should be made and published with scientific precision, and be such as to command public confidence. The meteorological departaent, he points out, besides furnishing science with valuable data from a locality well suited for the observation and collection of facts appertaining to certain phenomena of special interest, cannot fail to have a direct and practical value by affording security, by its predictions and weather-warnings, to life and property in seas navigated by vast numbers of native and foreign vessels, but subject, during four or five months of the year, to rotatory storms of appalling violence and danger. Its operations, as well as its purpose, would be twofold. There would be the systematic observation and record of the ordinary phenomena of pressure, temperature, humidity, rain, sunshine, wind, and hydrometeors, such as are usual in observatories of the first order, and observations of atmospheric electricity. The second branch would be the observation and collection, as far as possible, of facts and phenomena relating to typhoons, both for the protection of marine interests and for the purpose of contributing bit by bit to our knowledge of a subject at present but little understood. He recommends that measures should be taken to enable the director of the observatory to be placed in prompt telegraphic communication with Manila, Amoy, Shanghai, Saigon, and Singapore, as well as with the south of Japan, and indicates the steps necessary for this purpose.

As for terrestrial magnetism, the character of the rocks and soil of Hongkong, and the neighbouring promontory of Kau-lung, is not, he thinks, the most favourable that could be chosen for magnetic research, yet the position of the colony on the magnetic chart, and with respect to other places where observatories are already established, leaves no room to doubt the value of well-conducted observations.

As to the staff of the observatory, a director, Major Palmer very properly suggests, should be obtained through the Astronomer-Royal, and thinks a salary of not less than $700 l$. a year should be given. Minor officers could be obtained among the non-commissioned officers of the Ordnance Survey Companies of the Royal Engineers. The prime cost he estimates at $6300 l$., and the 